\title{
Heart rate variability as a biomarker for epilepsy seizure prediction
}

\author{
Moridani MK, Farhadi H \\ Department of Biomedical Engineering, Tehran Medical Branch, Islamic Azad University, \\ Tehran, Iran.m.karimi@gmail.com
}

\begin{abstract}
OBJECTIVE: Epilepsy is a neurological disorder that causes seizures of many different types. Recent research has shown that epileptic seizures can be predicted by using the electrocardiogrami instead of the electroencephalogram. In this study, we used the heart rate variability that is generated by the fluctuating balance of sympathetic and parasympathetic nervous systems to predict epileptic seizures.

METHODS: We studied 11 epilepsy patients to predict the seizure interval. With regar tos the fact that HRV signals are nonstationary, our analysis focused on linear features in the time and frequency domain of HRV signal such as RR Interval (RRI), mean heart rate (HR), high-frequency (HF) $(0.15-0.40 \mathrm{~Hz})$ and low-frequency (LF) $(0.04-0.15 \mathrm{~Hz})$, as well as LF/HF. Also, quantitative analyses of Poincaré plot features (SD1, SD2, and SD1/SD2 ratio) were performed. HRV signal was divided into intervals of 5 minutes. In each segment linear and nonlinear features were extracted and then the amount of each segment compared to the previous segment using a threshold. Finally, we evaluated the performance of our method using specificity and sensitivity. RESULTS: During seizures, mean HR, LF/HF, and SD2/SD1 ratio significantly increased while RRI significantly decreased. Significant differences between two groups were identified for several HRV features. Therefore, these parameters can be used as a useful feature to discriminate a seizure from a non-seizure The seizure prediction algorithm proposed based on HRV achieved $88.3 \%$ sensitivity and $86.2 \%$ specificity.

CONCLUSION: These results indicate that the HRV signal contains valuable information and can be a predictor for epilepsy seizure. Although our results in comparison with EEG ares a little bit weaker, the recording of ECG is much easier and faster than EEG. Also, our finding showed the results of this study are considerably better than recent research based on ECG (Tab. 1, Fig. 10, Ref. 17). Text in PDF www.elis.sk.

KEY WORDS: epileptic seizure, heart rate variability, linear and non-linear analysis, prediction.
\end{abstract}

\section{Introduction}

Epilepsy refers to chronic disorders and brain dysfunction in which normal communication between nerve cells in the brain is disturbed. Seizures are a sudden surge of electrical activity in the brain. Seizures occur alternately at intervals in the brain. Indeed, seizures depend on the location of epileptic discharges in the brain as well as symptoms and type of epileptic seizures. There are different types of seizures, which are associated with various risk factors for the patients depending on type and severity of epilepsy. Partial epilepsy is caused by a damage to one side of the brain, which is divided into two categories: simple partial epilepsy and simple complex epilepsy. Pure epilepsy lasts less than a minute. The symptoms depend on the location of epileptic discharges. Complex epilepsy lasts one to two minutes. Generalized epilepsy affects both sides of the brain and the affected individual suddenly becomes unconscious. Sympathetic nervous system dysfunction

Department of Biomedical Engineering, Tehran Medical Branch, Islamic Azad University, Tehran, Iran

Address for correspondence: M.K. Moridani, No. 29, Floor 4, Farjam St., Tehran-Pars, Tehran, Iran.

Postal Code: 1653989618

Phone: +982177874289 , Fax: +982188675452 occur in epilepsy with febrile seizure. A part of the body shakes uncontrollably in focal epilepsy, which may spread to other areas of the body and the whole body may shake uncontrollably. However, the affected individual does not lose consciousness completely.

An epileptic patient may be affected by two different kinds of seizures such as unilateral and bilateral seizures depending on the type of epilepsy. In single seizures, electrical discharge affects some areas of the brain (cortex), but the affected individual does not lose consciousness immediately. This type of seizure is called focal sensory epilepsy. In the bilateral assault, both hemispheres are simultaneously affected, and the affected individual loses consciousness immediately.

An algorithm that can predict the occurrence of epileptic seizures extremely ensures the patients and their caregivers of prognosis of epileptic seizures to either prevent irreparable damage to the patients or take necessary measures to reduce the severity of damages.

Electroencephalogram (EEG) signals are a convenient and well-known method for examining epileptic seizures. EEG refers to either superficial (noninvasive) or invasive records of brain activities. Needle electrodes are implanted in the scalp in the invasive method. Given that the brain is the origin of epileptic seizures recording EEG signal in epileptic patients is problematic and not easily accessible. Epileptic seizures also affect the autonomic 
nervous system and consequently activities of both sympathetic and parasympathetic nerves. Thus, the heart is one major organ affected by epileptic seizures. In this paper, ECG signals were used to predict epileptic seizures, which can be done more easily and more quickly. It is expected that the results of ECG recordings do not differ from the results of EEG signals. Hopefully, ECG signals can be used as a complementary approach to prediction of epileptic seizures. Finally, a combination of two EEG and ECG signals can help to improve the results and efficiency of the algorithm.

Many studies were performed to predict epileptic seizures. However, EEG signals were used in most of these studies. Martinerie et al covered 19 seizures in 11 patients in 1998. Density correlation method was utilized in the recent survey in which sensitivity was reported as $89 \%$ (1).

Le Van Quyen et al used EEG signals to predict 11 epileptic seizures in 9 patients in 2000. In the above study, similarity index algorithm was used with $94 \%$ sensitivity (2). Navaro et al used similarity index method to predict 41 epileptic seizures in 11 patients in 2002. They obtained sensitivity of $83 \%$ (3). Netoff T et al used power spectral density method to predict 45 seizures in 9 patients in 2009. They obtained sensitivity of $77.8 \%$ (4).

Yun Park et al used power spectral density method for prediction of 80 seizures in 18 patients in 2011 . They obtained sensitivity of $92.5 \%$ (5). The results of the recent study showed that epileptic patients are more prone to abnormal heart rhythms not only during the seizure but also before the seizure (6).

In general, epileptic seizures are associated with an apparent increase in heart rate in most cases. However, arrhythmia occurs, and heart rate decreases in some cases such as partial epilepsy and generalized epileptic seizures (7-8). The article is organized as follows.

In the second section, used database and method to predict seizures with ECG signal processing metrics and evaluation methods are discussed. In the third section, the results of these methods are discussed. Discussion and conclusion are presented in the fourth section. In this section, a summary and comparison of the proposed approach with previous studied are shown. Work limitations are also discussed. Some recommendations are also given for future work.

\section{Materials and methods}

Heart rate fluctuates under the influence of sympathetic and parasympathetic nervous systems. Short-term and long-term changes in heart rate reflect the autonomic nervous system function (9).

A typical ECG tracing of the cardiac cycle (heartbeat) consists of a P wave (atrial depolarization), a QRS complex (ventricular depolarization), and a $\mathrm{T}$ wave (ventricular repolarization and a $\mathrm{U}$ wave. Each wave and the distances between them are related to different parts of the heart and can be used to assess cardiac health, but $\mathrm{R}$ wave is more significant than other waves, which indicates ventricular contraction or a heartbeat. $\mathrm{R}-\mathrm{R}$ interval is also called beat-to-beat or normal-to-normal (NN) interval, which represents time interval between heart beats (Fig. 1).

A change in cardiac signal during two consecutive beats is called heart rate variability (10). HRV signal shows different modes

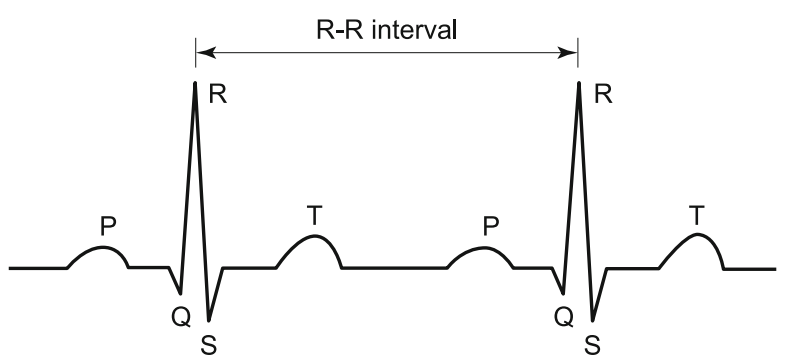

Fig. 1. ECG signal and $R-R$ intervals.

of cardiovascular diseases. Therefore, analysis of heart rate variability can be used as a tool to monitor changes in the function of the autonomic nervous system. However, less variable heart beats show relatively low health. Naturally, heart rate variability is directly related to individual health and healing. Increased heart rate variability increases individual health (11).

Studies have shown that correct information on autonomic nervous system function can be obtained using noninvasive HRV signal analysis in all brain disorders resulting from sympathetic and parasympathetic imbalance. HRV signal is a multivariate variable of cardiovascular systems, which represents dynamic characteristics, short-term and long-term correlations and complexities of the cardiac signal and autonomous nervous system. Nowadays, there are different methods for HRV signal processing, which can be divided into linear and nonlinear methods.

\section{Used database}

The data on partial epileptic patients available in Physionet Database is used in this study (12). Seven patients were examined in this study. In total, 11 seizures were observed during hospitalization of the patients. ECG signals with $200-\mathrm{kHz}$ frequency sampling, 12-bits per sample and 5-mV resolution were digitalized and recorded in epileptic patients. Seizure interval was specified in all patients and labeled in the database.

\section{Extracting linear and nonlinear features of HRV signal}

Changes are calculated with linear statistical methods, which are divided into time-domain and frequency-domain methods. A simple calculation is one main advantage of these features. However, statistical properties depend on the quality of recorded data. This quality may be affected by environmental noises. In timedomain method, R-R intervals show, analyze and examine high frequency changes or short-term changes in which various features can be extracted as follows: R-R mean interval, standard deviation of NN intervals (SDNN), Root Mean Square of the Successive Differences (RMSSD), the number of differences in consecutive R-R intervals greater than $50 \mathrm{~ms}$ (NN50), the ratio obtained by dividing total number of NN50 intervals to R-R (pNN50). Analysis of heart rate variability in adults showed that range of HRV signal consists of three frequencies as follows: low frequency (LF) (0.04-0.1 $\mathrm{Hz})$, high frequency $(\mathrm{HF})(0.15-0.4 \mathrm{~Hz})$ and very low frequency (VLF) $(0.0001-0.04 \mathrm{~Hz})(13)$. Fluctuations in these two components represent sympathetic and parasympathetic activity. In the 


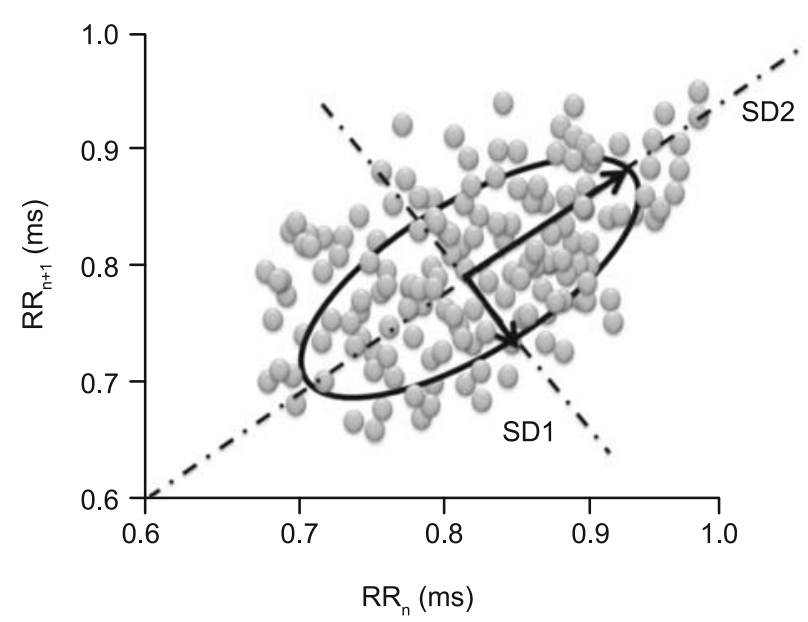

Fig. 2. Poincaré return map of RR intervals for a healthy individual $(\mathbf{n}=\mathbf{1 0 0})$.

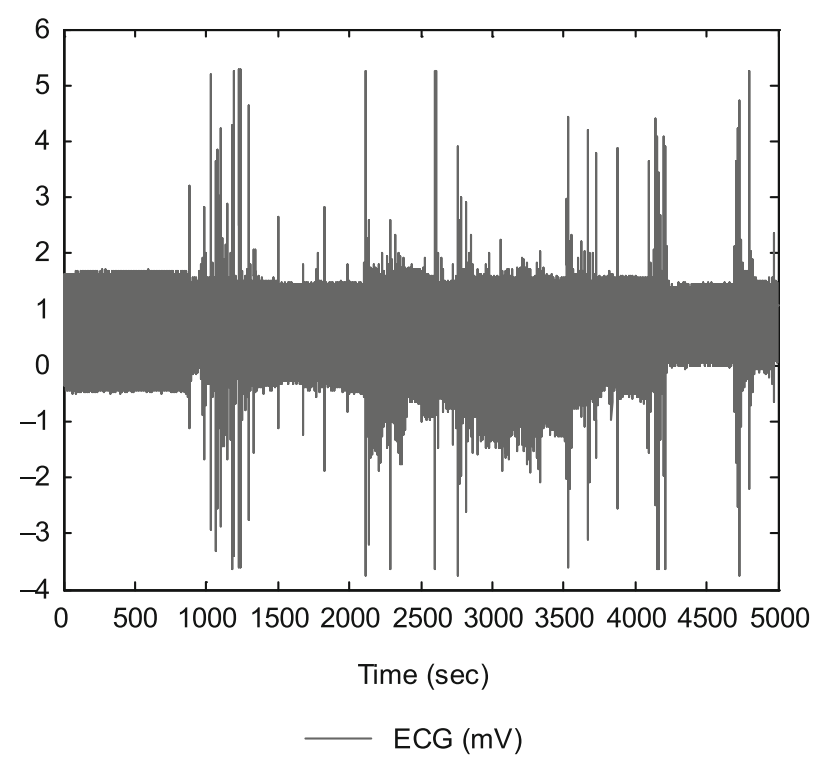

Fig. 3. ECG signal in an epileptic patient.

frequency-domain method, the ratio of these two components is used as a measure of the balance of sympathetic and parasympathetic function. In the frequency-domain method, VLF, LF, HF, LF / HF parameters can be extracted from HRV signal. In fact, calculated energy is different in various frequency bands (14).

It should be noted that properties of complex systems are ignored or deleted in linear methods, which cause an additional error. Physiological systems are fundamentally and inherently nonlinear. Since standard parameters in HRV only describe linear and periodic behaviors analysis, more complex and nonlinear relationship cannot be detected. Recent advances in the theory of nonlinear dynamics have facilitated signal analysis in nonlinear living organisms.

Nowadays, nonlinear techniques can describe processes in living biological organisms.
Poincaré return map is a relatively new technique for analyzing nonlinear dynamics such as HRV signal. Each point in the graph is specified as $\mathrm{n}=1,2,3, \ldots, \mathrm{k}\left(R R_{n}, R R_{n+1}\right)$ where $\mathrm{k}$ represents signal strength (15). Statistically, this mapping graphically displays the correlation between consecutive $\mathrm{R}-\mathrm{R}$ intervals. The mapping gives useful information on short-term and long-term fluctuations. The mapping is shown in Figure 2. $\mathrm{SD}_{1}$ and $\mathrm{SD}_{2}$ parameters are shown in this diagram. $\mathrm{SD}_{1}$ shows beat-to-beat rapid changes, which is more related to respiratory sinus arrhythmia. However, $\mathrm{SD}_{2}$ describes long-term beat-to-beat changes. $\frac{\mathrm{SD} 2}{\mathrm{SD} 1}$ is calculated to describe the relationship between these components (16). $\mathrm{SD}_{1}$ and $\mathrm{SD}_{2}$ values in Poincare mapping directly depend on statistical values of standard deviation of heart rate signal and two consecutive intervals of $\mathrm{R}$ peaks, which are calculated using equation (1) where $R R_{n+1}$ refers to the $(n+1)$ th beat-to-beat interval, $R_{n}$ represents to ${ }^{\text {th }}$ beat-to-beat interval and SD denotes standard deviation.

$$
\mathrm{SD}_{2}=\sqrt{2 \times \mathrm{SD}\left(\mathrm{RR}_{1}\right)^{2}-0.5 \times \mathrm{SD}\left(\mathrm{RR}_{\mathrm{n}+1}-\mathrm{RR}_{\mathrm{n}}\right)}
$$

\section{Prediction algorithm}

After recording ECG signals in epileptic patients, R peaks were detected in these signals using the method proposed by Pan and Tompkins (17). Then, R-R intervals were calculated, and HRV signals were organized by identifying the location of the peaks.

Figure 3 shows ECG signal in an epileptic patient recorded in about 83 minutes and 20 seconds. The patient experienced epileptic seizures from the 14th minute and 36th second to the 16th minute and 12 th second. In other words, seizures lasted from the 972nd second to 876th second. Figures 4 and 5, respectively, show ECG signal in an epileptic patient in a seizure interval and HRV signal in periods before, during and after the seizure.

Since location and duration of the seizure were specified in available data, HRV signal was divided into time intervals using

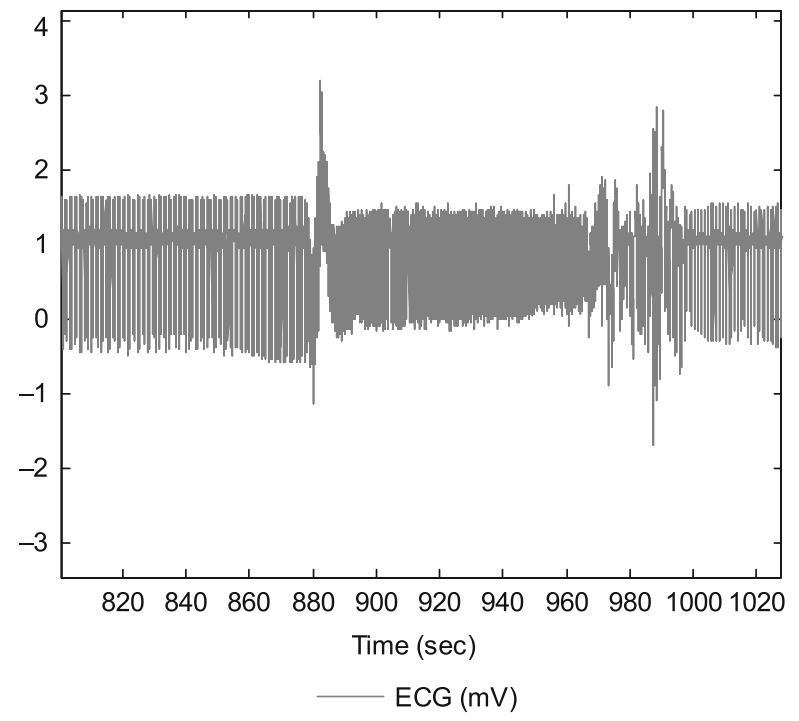

Fig. 4. Seizure interval in an epileptic patient. 


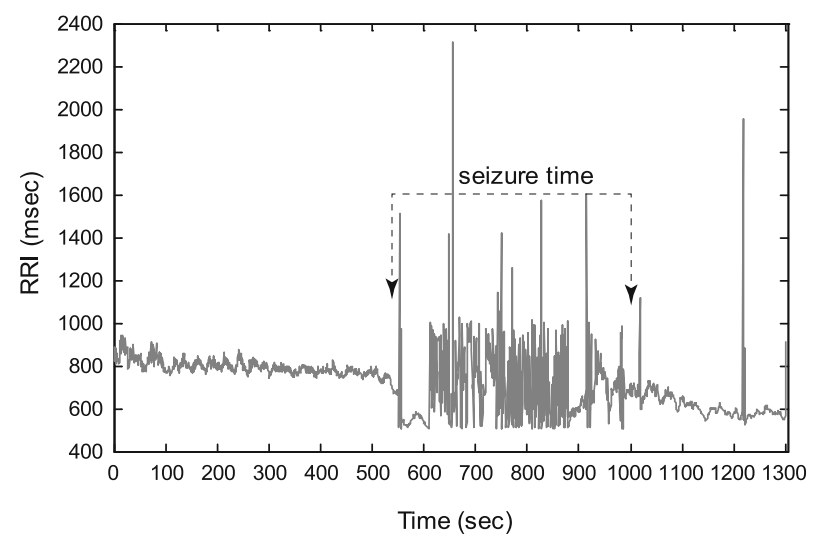

Fig. 5. HRV signal in epileptic patients before, during and after the seizure.

window function. Then, linear and nonlinear properties were extracted in each interval in the signal. Paired t-test was used to detect those features that were significantly different in this interval compared to the previous interval. The detected intervals were used to predict epileptic seizures. Figure 1 shows a diagram of the proposed algorithm.

\section{Selecting an optimal threshold}

Threshold values with the best optimum sensitivity and the least prediction error were chosen. In cases where specificity (predicting non-seizure interval) was acceptable for different thresholds, the threshold with the lowest prediction error would be selected. An alarm is given when the desired feature reaches the threshold. Given that the threshold is different for each patient, a single threshold is selected for each patient. For example, mean and variance values of nth window is half the average values of (n-1)th window. First, specificity and initial sensitivity values were calculated. Then, sensitivity values and the rate of incorrect pre-

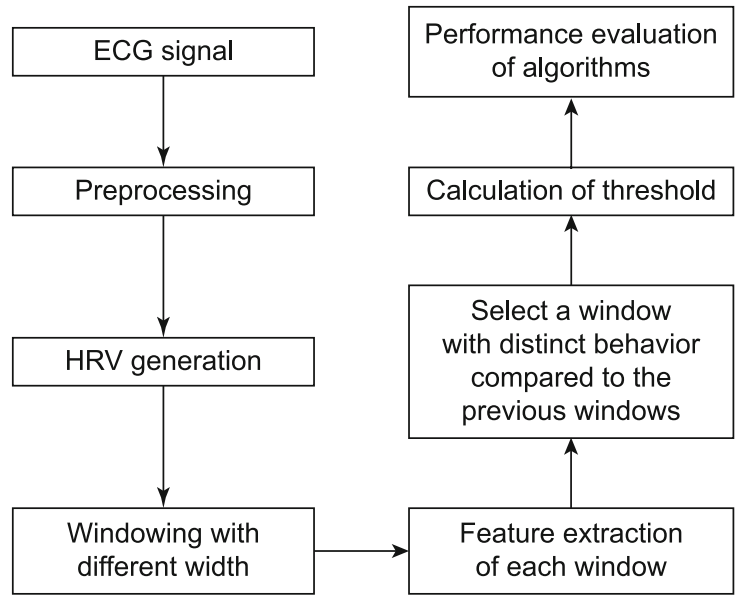

Fig. 6. An algorithm to determine a threshold to the prediction of the future condition of the patient.
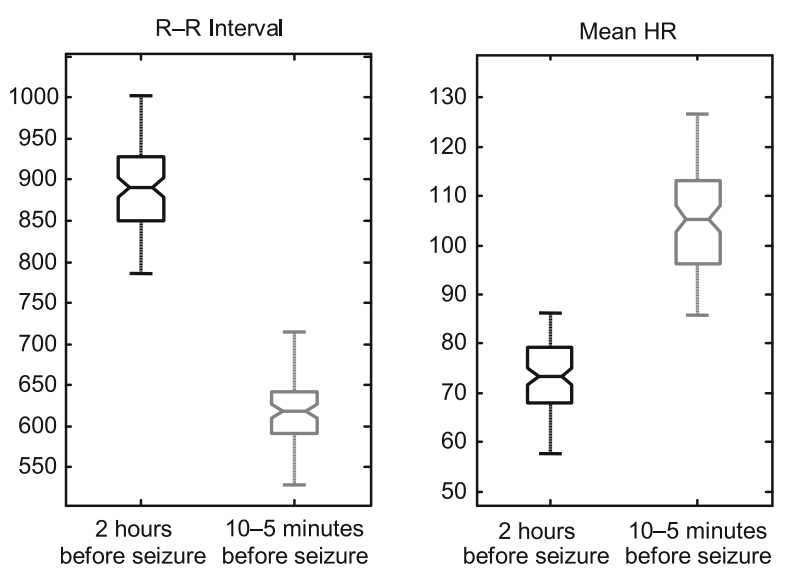

Fig. 7. Comparison of R-R Interval and mean heart rate in Poincare plot in the seizure and non-seizure interval.
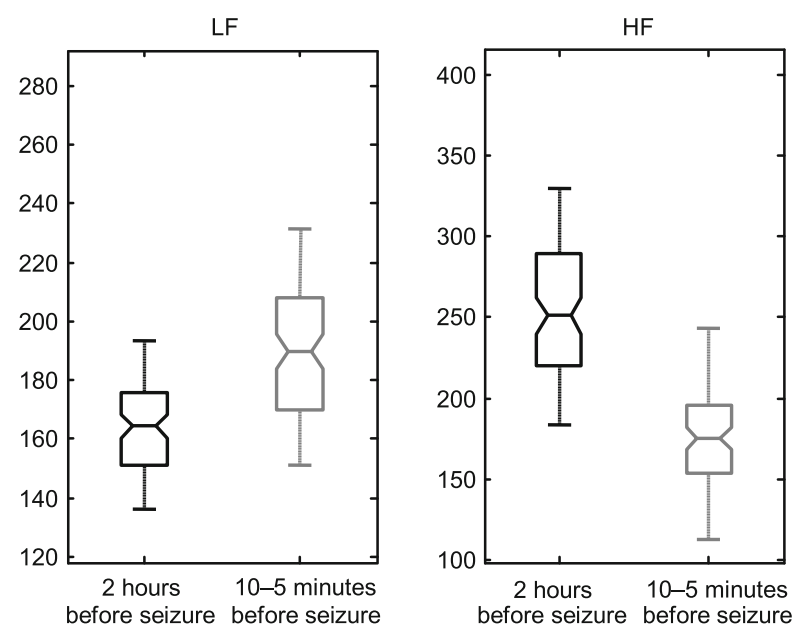

Fig. 8. Comparison between low-frequency and high-frequency in Poincare plot in the seizure and non-seizure interval.

dictions have been computed by changing the threshold. Finally, the best threshold with the highest specificity and maximum sensitivity and the least prediction error were selected.

\section{Introducing prediction criteria}

In this paper, several features were used to predict epileptic seizures in patients. The goal of all these features lied in achieving optimum results to obtain complete information on the future status of patients for nurses and practitioners. Therefore, it is essential to define some criteria in this area.

An important criterion is the false positive rate or false-alarm rate, which shows how much the right time of the epileptic seizure was predicted in the proposed system or algorithm. In other words, lower criteria represent the higher efficiency of the algorithm in the prediction. The second criteria are prediction of the horizon, which shows the time of alarm to nurses and practitioners and specifies how long before the seizure the alarm is given. The greater the prediction horizon, the greater the efficiency of the system, so 

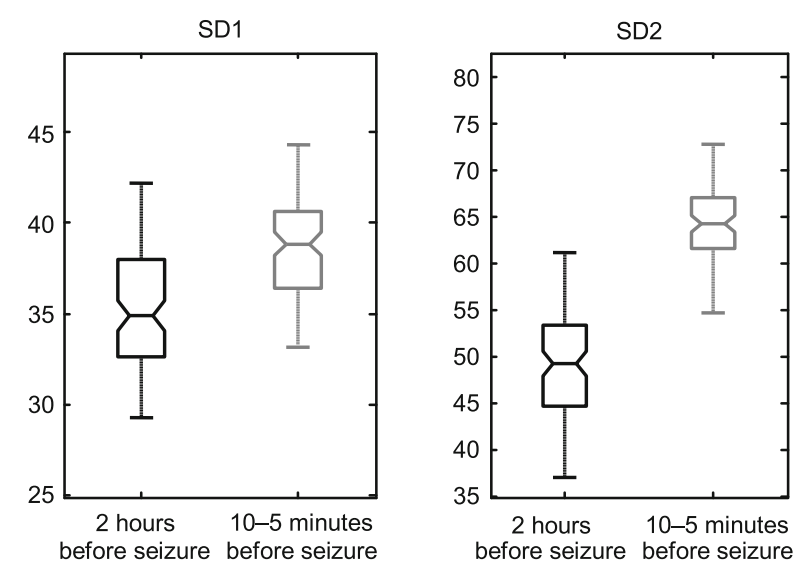

Fig. 9. Comparison between SD1 and SD2 in Poincare plot in the seizure and non-seizure interval.
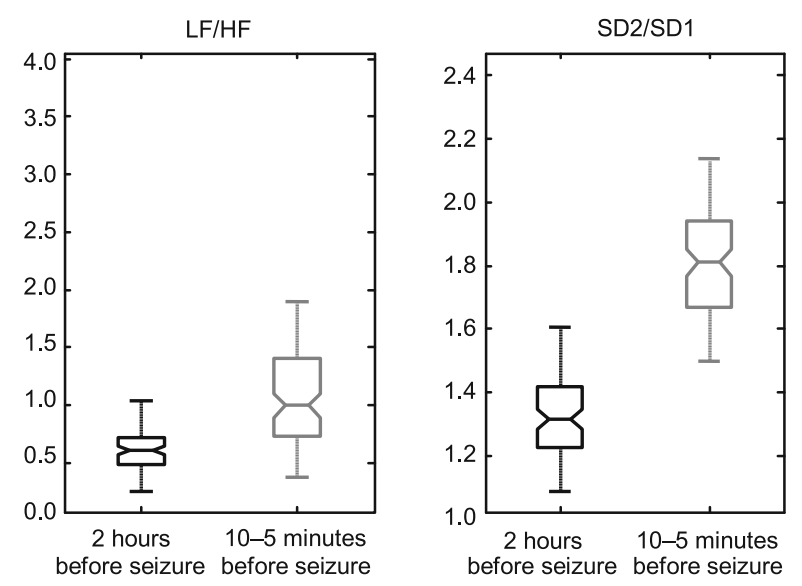

Fig. 10. Comparison between low-frequency to high-frequency ratio and SD2 to SD1 ratio in Poincare plot in the seizure and non-seizure interval.

that nurses and practitioners would have enough time to provide more facilities and care measures for the patients (Figs $6-10$ ).

\section{Statistical test}

In general, statistical tests aim to determine criteria for the feature or features extracted from the signals recorded from the patients. This paper aimed to examine the features extracted from HRV signal at different time intervals. Thereby, paired sample ttest was used to determine changes and distinction in extracted features at various time intervals. Paired t-test also called before and after test aimed to compare means in the two groups. The assumption of normality of variables in the two groups should be observed before the test. There are two observations (observations close to seizure and intervals far away from the seizure) for each in this test.

In the output of the test, mean and standard deviation of the variables are shown to describe the data. Then, the results of correlation between values close to seizure and far away from the seizure (this part does not affect the interpretation of mean com- parison results) are given. Then, the results of mean equality in the two groups by the mean difference in the two intervals with zero value are presented. If significance values were less than $\alpha$, the assumption of equality of means in the two groups would be rejected at $\alpha$ error level. In this study, the significance level was considered less 0.5 .

\section{Evaluation of the performance of the proposed algorithm}

To evaluate the performance of the proposed algorithm, we introduced two criteria, namely, sensitivity and specificity. The sensitivity $\left(\mathrm{S}_{\mathrm{n}}\right)$ of seizure detection is the probability that the detection is positive when the HRV segments are with the seizure.

The specificity $\left(\mathrm{S}_{\mathrm{p}}\right)$ is defined as the probability that the seizure detection result says a non-seizure segment, when in fact, they are seizure free. The sensitivity and specificity measures are given in equation (2). True positive (TP): Sick people correctly identified as sick, False positive (FP): Healthy people incorrectly identified as sick, True negative (TN): Healthy people correctly identified as healthy and False negative (FN): Sick people incorrectly identified as healthy.

$$
\begin{aligned}
& \text { Sensitivity }\left(\mathrm{S}_{\mathrm{e}}\right)=\frac{\mathrm{TP}}{\mathrm{TP}+\mathrm{FN}} \times 100 \\
& \text { Specificity }\left(\mathrm{S}_{\mathrm{p}}\right)=\frac{\mathrm{TN}}{\mathrm{TN}+\mathrm{FP}} \times 100
\end{aligned}
$$

\section{Results}

In order to observe changes in cardiac signal of epileptic patients, linear and nonlinear values mentioned in the previous section at following intervals were calculated: ten to five minutes before the seizure, from fifteen to ten minutes prior to seizure , from twenty to fifteen minutes before the seizure and a distant interval from the seizure range (two hours before the seizure ).

Table 1 shows a qualitative (statistical) comparison of linear and nonlinear features using paired t-test and calculated $p$-value at two-time intervals ranging from ten to five minutes before the seizure and two hours before the seizure. It can be observed that RRI dronped in moments close to the seizure, but Mean HR, $\frac{\mathrm{LF}}{\mathrm{HF}}$ and $\frac{\text { SD2 }}{\text { SD1 }}$ and significantly increased in most intervals compared to the two hours before the seizure. Since p-values for RRI, Mean $\mathrm{HR}, \frac{\mathrm{LF}}{\mathrm{HF}}$ and $\frac{\mathrm{SD} 2}{\mathrm{SD} 1}$ features were less than 0.01 , it can be concluded that these features have acceptable prediction values and can be regarded as appropriate criteria for predicting the future condition of the patients.

Also, examining behavioral features extracted at different time intervals reflects changes in the interval of about 30 minutes before the seizure but most changes occurred in the interval of 15 minutes before the seizure. Therefore, the intervals of 10-15 minutes and 5-10 minutes before the seizure were more emphasized than other intervals and HRV signal features were more studied in the previous intervals than the last intervals (Tab. 1).

Two ratios of $\frac{\mathrm{SD} 2}{\mathrm{SD} 1}$ and $\frac{\mathrm{SD} 2}{\mathrm{SD} 1}$ showed identical behaviors, which confirmed a significant correlation between these two features since these two indicate parasympathetic and sympathetic activities. Any increase or decrease in these two features is due to different 
Tab. 1. Comparison of linear and non-linear features between 10-5 min and 2 hours before seizure.

\begin{tabular}{lccc}
\hline Feature type & $\begin{array}{c}2 \text { hours before } \\
\text { seizure }\end{array}$ & $\begin{array}{c}10-5 \text { min before } \\
\text { seizure }\end{array}$ & $\mathrm{p}$ \\
\hline RRI $(\mathrm{ms})^{*}$ & $885.46 \pm 57.64$ & $614.21 \pm 43.81$ & 0.008 \\
\hline Mean HR & $73.43 \pm 8.58$ & $106.32 \pm 13.23$ & 0.006 \\
\hline LF & $163.14 \pm 19.6$ & $190.09 \pm 26.21$ & 0.34 \\
\hline HF & $256.28 \pm 44.57$ & $177.63 \pm 31.72$ & 0.028 \\
\hline LF/HF* & $0.64 \pm 0.23$ & $1.07 \pm 0.56$ & 0.009 \\
\hline SD1 & $35.56 \pm 4.82$ & $39.12 \pm 3.08$ & 0.36 \\
\hline SD2 & $50.44 \pm 7.67$ & $65.12 \pm 4.19$ & 0.0025 \\
\hline SD2/SD1 $*$ & $1.33 \pm 0.13$ & $1.80 \pm 0.22$ & 0.008 \\
\hline
\end{tabular}

extraction method. Thus, it can be concluded that HRV signal behavior before 20-5 minutes interval prior to seizure is suitable for extraction of the features with regard to comparable intervals in which maximum changes were observed in the extracted features.

To determine the threshold for the seizure predicting measurement system, the best feature to investigate HRV signal behavior at various time intervals with significant distinction were selected. These features have been chosen using paired t-test with a p-value less than 0.01 .

The threshold values with the best sensitivity and the least prediction error have been selected. In cases where acceptable specificity for different thresholds (predicting noninvasive interval) were obtained, the threshold with the least prediction error has been determined. An alarm is given when the threshold reaches the desired feature. Given that the threshold is different for each patient, a single threshold should be considered for each patient. For example, mean and variance values of nth window were half the average values of (n-1)th window. First, initial values of specificity and sensitivity were calculated. Then, sensitivity value and the rate of incorrect predictions were determined for other thresholds by changing the threshold. Finally, the best threshold values with the maximum specificity and high sensitivity and the least false predictio rate were selected.

The results generated based on thresholding method show that it is able to distinguish high and low risk episodes and the seizure prediction algorithm proposed based on HRV achieved $88.3 \%$ sensitivity and $86.2 \%$ specificity.

\section{Conclusion}

This study analyzed 8 HRV features to predict epileptic seizures from clinical data collected from 7 patients. The analysis results showed that HRV features, such as mean HR, $\frac{\mathrm{SD} 2}{\mathrm{SD} 1}$ and $\frac{\mathrm{LF}}{\mathrm{HF}}$ changed 5-10 minutes before seizure onset in all seizure episodes. The possibility of realizing a HRV-based seizure prediction system was shown through these analyses.

Our data showed that epileptic seizures are associated with increased heart rate, indicating an increased sympathetic tone and reduced vagal tone. HRV parameter changes are more difficult to interpret and need further investigation. We credit that these features are useful biomarkers for clinical use. Now, we are in the process of working with physicians to find out more fea- tures for detection and prediction of epileptic seizures with HRV signal information.

\section{References}

1. Martinerie J, Adam C, Le Van Quyen M, Baulac M, Clemenceau S, Renault B, Varela F. Epileptic seizures can be anticipated by non-linear analysis. Nat Med 1998; 4: 1173-1176.

2. Le van Quyen M, Adam C, Martinerie J, Baulac M, Clemenceau S, Varela F. Spatio-temporal characterizations of nonlinear changes in intracranial activities prior to human temporal lobe seizures. Eur J Neurosci 2000; 12: 2124-2134.

3. Navaro V, Martinerie J, Le Van Quyen M, Clemenceau S, Adam C, Baulac M, Varela F. Seizure anticipation in human neocortical partial epilepsy. Brain 2002; 125: 640-655.

4. Netoff T, Park Y, Parh K. Seizure prediction using cost-sensitive support vector machine. In: Proceedings of 31 st Annual International Conference of the IEEE EMBS Minneapolis, Minnesota, USA, pp. 3322-3325, 2009.

5. Park Y, Luo L, Parhi KK, Netoff T. Seizure prediction with the spectral power of EEG using cost-sensitive support vector machine. Epilepsia 2011; 5 (10): 1761-1770.

6. Malarvili MB, Mesbah M, Boashash B. Time-Frequency Analysis of Heart Rate Variability for Neonatal Seizure Detection. EURASIP Journal on Advances in Signal Processing, pp. 50396- 50406, 2007.

7. Harnod T, Yang CC, Hsin YL, Wang PJ, Shieh KR, Kuo TB. Heart rate variability in patients with frontal lobe epilepsy. Seizure 2009; 18 (1): $21-25$.

8. Britton JW, Ghearing GR, Benarroch EE, Cascino GD. The ictal bradycardia syndrome: Localization and lateralization. Epilepsia 2006; 47: 737-744.

9. Malik M. Heart Rate Variability:Standards of Measurement, Physiological Interpretation, and Clinical Use. Ann Noninvas Electrocardiol 1996; 1: 151-181.

10. Gang Y, Malik M. Heart Rate Variability Analysis in General Medicine. Id Pacing Electrophysiol J 2003; 3 (1): 34-40.

11. Khazan IZ. Heart rate variability. The clinical handbook of biofeedback: A step by step guide for training and practice with mindfulness. John Wiley \& Sons publication, 2013, 97-117.

\section{2. http://physionet.org/physiobank/database/szdb}

13. Berntson GG, Bigger JT, Eckberg DL,Grossman P, Kaufmann PG, Malik M. Heart rate variability:origins, methods, and interpretive caveats. Psychophysiology 1997; 34: 623-648

14. Malliani A, Pagani M, Lombardi F, Cerutti S. Cardiovascular, neural regulation explored in the frequency domain. Circulation 1991; 84: 482-492.

15. Lerma C, Infante O, Grovas H, Jose MV. Poincare' plot indexes of heart rate variability capture dynamic adaptations after haemodialysis in chronic renal failure patients. Clin Physiol Func Im 2003; 23: 72-80.

16. Toledo E, Gurevitz O, Hod H, Eldar M, Akselrod S. Wavelet analysis of instantaneous heart rate: a study of autonomic control during thrombolysis. Am J Physiol Regul Integr Comp Physiol 2003; 284: 1079-1091.

17. Pan J, Tompkins WJ. A real-time QRS detection algorithm. IEEE Transact Biomed Engineer 1985; 32: 230-236.

Received August 8, 2016. Accepted October 27, 2016. 